\title{
Détermination rapide de l'extrait sec des fromages à l'aide d'un four à micro-ondes
}

\author{
par
}

\author{
A. DASEN et R. GRAPPIN
}

Mots clés :

Four à micro-ondes - Dosage - Extrait sec - Fromage.

Titre abrégé :

Extrait sec de fromage par four à micro-ondes.

\section{S u m m a ry}

RAPID MICROWAVE OVEN DETERMINATION OF THE DRY MATTER CONTENT IN CHEESE

An experiment was carried out to determine the repeatability and accuracy of the dry matter determination of cheese with the microwave oven instrument TMS Checker K375 A (Anritsu Co. Japon).

Sixty one samples of various type of cheeses (fresh lactic cheese, soft cheese semi-hard and hard cheese) ranging from $19.9 \%$ to $62.8 \%$ dry matter, were analysed with the microwave oven (4 replicates) and with the reference drying oven method (2 replicates).

The coefficient of variation of repeatability is on an average $0.26 \%$ for the microwave and varies from $0.28 \%$ for high moisture content cheese to $0.10 \%$ for low moisture content cheese, for the reference method.

The standard deviation of accuracy of the microwave oven, calculated from the algebraic mean differences between the two methods is $0.27 \%$ dry matter content, indicating a $95 \%$ confidence interval of $\pm 0.54 \%$ for the estimated true value.

In a field experiment, it has been demonstrated that the TMS Checker is a useful tool for checking rapidly the variation of soft Key words:

Microwave oven - Determination - Moisture - Cheese.

I.N.R.A., Station Expérimentale Laitière - 39800 Poligny. 
cheese moisture during drainage. The dry matter content can be estimated from a single microwave determination with an overall accuracy of $\pm 0.68 \%$ dry matter for a $95 \%$ confidence interval.

\section{INTRODUCTION}

Pour déterminer la teneur en matière sèche des fromages, les laboratoires ont habituellement recours à la méthode classique de séchage à l'étuve à $102^{\circ} \mathrm{C}$ avec pesée du produit avant et après dessication. Cette méthode qui sert de référence a un temps de réponse de plusieurs heures, et ceci peut représenter un handicap important, notamment en technologie fromagère où il est utile de connaître rapidement l'extrait $\mathrm{sec}$, soit de la matière première mise en œuvre, soit du produit fini ou en cours de fabrication.

Devant ce besoin de rapidité de réponse, plusieurs solutions ont été envisagées : mesure de l'absorption infra-rouge par les composants du fromage à l'aide des appareils de type Milko-Scan ou MultiSpec (Biggs, 1979), mesure de la réflectance dans le proche infra-rouge avec des appareils tel que l'Infralyseur, ou, pour des fromages frais maigres, mesure de la viscosité apparente (Corrieu et al., 1982). Ces techniques sont encore au stade des études et, bien qu'elles soient précises, ont l'inconvénient de nécessiter des calibrages spécifiques souvent délicats à réaliser. De ce fait, la mesure de la perte de poids après dessication du produit est encore une technique attrayante en raison de sa simplicité et de son faible coût. Pour accélérer la dessication, des techniques de chauffage par rayonnement infra-rouge ont été préconisées, mais la précision serait limitée et dépendante de la teneur en extrait sec du produit analysé (Multon et al., 1981). Plus récemment, des fours utilisant les propriétés des micro-ondes sont apparus sur le marché; ils permettent de sécher rapidement le produit à analyser et, pour un certain nombre d'entre eux, de faire automatiquement les pesées. Nous donnons ici les résultats de plusieurs essais effectués avec un four à micro-ondes destiné à l'analyse du lait, le Total Milk Solids Checker (modèle K $375 \mathrm{~A}$ construit par la Société Anritsu, Japon) et portant sur plusieurs types de fromages. Cet appareil a déjà fait l'objet de plusieurs études de détermination de l'extrait sec du lait (Grappin et Jeunet, 1982 ; Hamada et al., 1977).

\section{MATERIELS ET METHODES}

\section{a) Principe du four à micro-ondes}

Dans les fours conventionnels, la source de chaleur émet des radiations infra-rouges qui sont absorbées par la surface du produit 
et la chaleur se transmet à l'intérieur du produit par simple conduction, nécessitant un temps de chauffage plus ou moins long suivant le volume du produit.

Dans un four à micro-ondes, on utilise la propriété de l'eau d'absorber l'énergie électromagnétique transportée par les microondes et de la transformer en chaleur. La source de micro-ondes est un magnétron qui produit des radiations à $2450 \mathrm{MHz}$, soit une longueur d'onde moyenne de $2,2 \mathrm{~cm}$. L'eau étant une molécule polaire, l'action des micro-ondes entraîne des mouvements de vibration des molécules qui, en l'absence de frottement, se feraient à la même fréquence que celles des micro-ondes. Toutefois, les mouvements de vibrations sont gênés par la présence des autres molécules et il se produit un échauffement et une dessication du produit par friction moléculaire (Curnutte, 1980). L'énergie étant directement dispersée dans tout l'échantillon soumis aux radiations, le temps de chauffage est très réduit par rapport au chauffage par rayonnement.

\section{b) Appareil}

L'appareil TMS Checker K 375 A a déjà été décrit par Takahashi et al., (1978), toutefois, en raison de la grande diversité des appareils disponibles nous rappellerons brièvement les principales caractéristiques originales du TMS Checker. L'appareil comprend : un magnétron et un four guide d'onde rectangulaire et de petite dimension, terminé par un bain d'eau régulé à $40^{\circ} \mathrm{C}$ qui absorbe les radiations en excès évitant une réflexion des ondes sur les parois du four qui pourrait entraîner des zones de chauffage irrégulier et un échauffement du magnétron. A l'intérieur se trouvent deux plateaux, un plateau fixe sur lequel est placé la capsule avant analyse et le plateau de la balance. Les plateaux sont situés de façon à recevoir le maximum du champ électrique.

\section{c) Protocole expérimental}

L'appareil étant conçu pour avoir une puissance d'émission des micro-ondes constante, seul le couple poids de l'échantillon et temps de chauffage peut être modifié. Afin de définir ce couple, nous avons analysé au cours d'un essai préliminaire trois types de fromages en utilisant trois prises d'essai différentes de : 0,2 à $0,3 \mathrm{~g}$; de 0,4 à $0,5 \mathrm{~g}$ et de 0,75 à $1 \mathrm{~g}$. Pour chaque prise d'essai, nous avons appliqué quatre temps de chauffage : $2,3,4$ et $5 \mathrm{~min}$.

L'analyse statistique des résultats a montré qu'avec une prise d'essai de 0,4 à $0,5 \mathrm{~g}$ et un temps de chauffage de $4 \mathrm{~min}$, on obtenait la meilleure précision (répétabilité et justesse) des mesures pour les fromages analysés.

Pour effectuer les mesures, les échantillons de fromages sont râpés ou malaxés et disposés dans les capsules en verre. L'échan- 
tillon est enveloppé de papier en fibre de verre pour éviter la pro. jection de fromage pendant le chauffage.

\section{d) Fromages}

La teneur en extrait sec de 61 échantillons de fromages (5 échan. tillons de fromage frais, 10 de fromage à pâte molle, type carré de l'Est, 10 de fromage à raclette et 36 de gruyère de Comté d'âges différents) a été déterminée en quatre exemplaires à l'aide du four à micro-ondes et en double exemplaire par la méthode de référence à l'étuve (Norme AFNOR NF V 04-82). Les résultats sont exprimés en gramme d'extrait sec pour $100 \mathrm{~g}$ de fromage ( $\%$ extrait sec).

\section{e) Analyses statistiques}

Les valeurs de répétabilité et de justesse ont été déterminées selon les modèles décrits précédemment pour les méthodes indirectes d'analyse du lait (Grappin, 1976). La répétabilité (r) indique l'étroitesse de l'accord entre analyses d'un même échantillon. Par convention, elle est représentée par l'écart arithmétique entre 2 déterminations d'un même échantillon, ayant une probabilité de $95 \%$ de ne pas être dépassée, soit $r=2,77 . \sigma_{r}, \sigma_{r}$ étant l'écart-type de répétabilité. Dans cette étude $\sigma_{\mathrm{r}}$ est obtenu à partir de l'analyse de la variance (écart-type intra-échantillon) des résultats obtenus avec l'appareil pour chaque échantillon.

La répétabilité peut également être exprimée à l'aide du coefficient de variation $\mathrm{CV}=\left(\sigma_{\mathrm{r}} /\right.$ Extrait $\mathrm{sec}$ moyen $) \times 100$.

La justesse qui indique l'étroitesse de l'accord entre la méthode de référence et l'appareil est estimée globalement à l'aide de la moyenne et de l'écart-type des différences algébriques entre les deux méthodes calculées sur les moyennes de plusieurs déterminations par échantillon.

\section{RESULTATS ET DISCUSSION}

\section{a) Répétabilité}

Les calculs ont été faits pour chaque type de fromage et sont résumés dans le tableau 1 . On constate que l'écart-type de répétabilité de la méthode de référence est peu influencé par le niveau d'extrait sec du fromage, avec une valeur moyenne de $0,066 \%$ d'extrait sec. En revanche, l'écart-type varie proportionnellement avec l'extrait sec dans le cas du four à micro-ondes, passant de $0,054 \%$ d'extrait sec pour le fromage frais à $0,189 \%$ pour le Comté. Dans ce cas, il convient d'utiliser le coefficient de variation pour exprimer la répétabilité de l'appareil sur toute l'étendue des mesures, soit pour l'ensembe des fromages, un coefficient de variation moyen de $0,26 \%$ (en valeur relative du pourcentage d'extrait sec). 


\section{TABLEAU 1 - TABLE 1}

Répétabilité des méthodes de dosage de l'extrait sec (four à micro-ondes et méthode de référence)

Repeatability of methods for measuring dry matter (microwave oven and reference method)

\begin{tabular}{|c|c|c|c|c|c|c|}
\hline \multirow{2}{*}{$\begin{array}{l}\text { Type de fromage } \\
\text { Type of cheese }\end{array}$} & \multirow{2}{*}{$\begin{array}{l}\text { Nombre } \\
\text { d'échantil- } \\
\text { lons } \\
\text { Number } \\
\text { of } \\
\text { samples }\end{array}$} & \multirow{2}{*}{$\begin{array}{c}\text { E.S. } \\
\text { moyen (\%) } \\
\text { Mean of } \\
\text { dry matter } \\
(\%) \\
\bar{x}\end{array}$} & \multicolumn{2}{|c|}{$\begin{array}{l}\text { Micro-ondes } \\
\text { Microwave }\end{array}$} & \multicolumn{2}{|c|}{$\begin{array}{l}\text { Référence } \\
\text { Reference }\end{array}$} \\
\hline & & & $\sigma_{\mathrm{r}}$ & $\mathrm{CV}$ & $\sigma_{\mathrm{r}}$ & CV \\
\hline Fromage frais (fresh cheese) & 5 & 19,9 & 0,054 & 0,27 & 0,055 & 0,28 \\
\hline Pâte molle (soft cheese) & 10 & 47,8 & 0,124 & 0,26 & 0,076 & 0,16 \\
\hline Raclette (Raclette) & 10 & 57,4 & 0,126 & 0,22 & 0,074 & 0,13 \\
\hline Comté (Comté) & 36 & 62,8 & 0,189 & 0,30 & 0,061 & 0,10 \\
\hline \multicolumn{7}{|c|}{$\begin{array}{l}\sigma_{\mathrm{r}}=\text { Ecart-type de répétabilité exprimé en } \% \text { de l'extrait sec. } \\
\mathrm{CV}=\text { Coefficient de variation }=\left(\sigma_{\mathrm{r}} / \overline{\mathrm{x}}\right) 100 . \\
\sigma_{\mathrm{r}}=\text { Standard deviation of repeatability in } \% \text { of dry matter. } \\
\mathrm{CV}=\text { Coefficient of variation }=\left(\sigma_{r} / \bar{x}\right) 100 .\end{array}$} \\
\hline
\end{tabular}

Soulignons que la répétabilité des deux techniques est la même dans. le cas de l'analyse des fromages à faible extrait sec (fromage frais) et environ trois fois moins bonne pour le four à micro-ondes dans le cas des fromages à pâte cuite à $62,8 \%$ d'extrait sec.

Pour des fromages avoisinant 60 à $62 \%$ d'extrait sec, Pieper et al., (1977) ont obtenu au cours d'un essai interlaboratoire avec un autre type d'appareil des écarts-types de répétabilité un peu meilleurs variant de 0,071 à $0,097 \%$ d'extrait sec.

Shanley et Jameson (1981) ont obtenu des résultats très variables en analysant une grande variété de fromages à l'aide d'un four conventionnel aménagé. Ils ont obtenu en particulier des écartstypes de répétabilité de $0,31 \%$ d'extrait sec pour des fromages de Camembert et $0,16 \%$ pour des fromages de type suisse.

\section{b) Justesse}

Pour l'ensemble des 61 fromages analysés, la relation entre les résultats micro-ondes $(\mathrm{x})$ et ceux de la méthode de référence (y) 
TABLEAU 2 - TABLE 2

Justesse des mesures d'extrait sec (en \%) obtenues avec le four à micro-ondes après calibrage de l'appareil

Accuracy of the mean of the dry matter determinations (in \%) obtained with the microwave oven after calibration of the instrument

\begin{tabular}{|c|c|c|c|c|c|}
\hline \multirow{2}{*}{$\begin{array}{l}\text { Type de fromage } \\
\text { Type of cheese }\end{array}$} & \multirow{2}{*}{$\begin{array}{c}\text { Nombre } \\
\text { d'échantillons } \\
\text { Number of } \\
\text { samples }\end{array}$} & \multirow{2}{*}{$\begin{array}{l}\text { E.S. } \\
\text { moyen } \\
\text { Mean dry } \\
\text { matter }\end{array}$} & \multicolumn{3}{|c|}{$\begin{array}{c}\text { Différences moyennes micro-ondes } \\
\text { Référence } \\
\text { Mean differences microwave } \\
\text { Reference }\end{array}$} \\
\hline & & & $\begin{array}{l}\text { Moyenne } \\
\text { Mean }\end{array}$ & $\begin{array}{l}\text { Ecart-type } \\
\text { Standard- } \\
\text { deviation }\end{array}$ & $\begin{array}{l}\text { Coefficient } \\
\text { de variation } \\
\text { Coefficient } \\
\text { of variation }\end{array}$ \\
\hline Fromage frais (fresh cheese) & 5 & 19,9 & $+0,34$ & 0,09 & 0,43 \\
\hline Pâte molle (soft cheese) & 10 & 47,8 & $-0,20$ & 0,10 & 0,21 \\
\hline Comté $(20$ h) (Comté $[20$ hours $]$ ) & 12 & 61,7 & $+0,28$ & 0,24 & 0,38 \\
\hline Comté (1 mois) (Comté [1 month]) & 12 & 62,6 & $-0,09$ & 0,29 & 0,47 \\
\hline Comté ( 3 mois et plus) (Comté [3 months and over]) & 12 & 64,2 & $-0,09$ & 0,14 & 0,22 \\
\hline
\end{tabular}


est donnée par l'équation de régression suivante : $\mathrm{y}=1,00489 \mathrm{x}-$ 0,41 avec un coefficient de corrélation linéaire de 0,9998 et un écarttype résiduel de $0,27 \%$ d'extrait sec; ces valeurs sont calculées à partir des moyennes des résultats d'analyses faites sur chaque échantillon. La valeur du coefficient de régression n'est pas statistiquement différente de l'unité $(b=1,00489 \pm 0,00543)$ pour une probabilité de $95 \%$. Par conséquent, il n'est pas nécessaire d'appliquer une correction de pente, seule une correction moyenne de calibrage de l'appareil (de -0,14 dans le cas présent), est suffisante pour tenir compte de la différence moyenne des résultats entre le four à micro-ondes et méthode de référence.

Dans le tableau 2, nous avons porté les paramètres de justesse de l'appareil, c'est-à-dire les moyennes et écarts-types des écarts entre la méthode par micro-ondes et la méthode de référence pour chaque type de fromage. On constate une amplitude des moyennes des écarts assez grande (de $+0,34$ d'extrait sec pour les fromages frais à $-0,20$ pour les pâtes molles) et des écarts-types plus faibles pour les fromages les plus humides.

L'analyse de variance (tab. 3) de ces différences montre une influence hautement significative du type de fromage sur la moyenne des écarts. Cela signifie que pour obtenir des résultats plus justes, des corrections de calibrage égales aux moyennes des écarts portées

\section{TABLEAU 3 - TABLE 3}

Analyse de la variance des différences moyennes entre micro-ondes et méthode de référence

Analysis of variance of the mean differences between microwave and reference methods

\begin{tabular}{l|c|c|c|c}
\hline \multicolumn{1}{c|}{$\begin{array}{c}\text { Source de variation } \\
\text { Origin of variation }\end{array}$} & $\begin{array}{c}\text { Somme des } \\
\text { carrés des } \\
\text { écarts } \\
\text { Sum of } \\
\text { squares }\end{array}$ & $\begin{array}{c}\text { Degré de } \\
\text { liberté } \\
\text { Degree of } \\
\text { freedom }\end{array}$ & $\begin{array}{c}\text { Carré } \\
\text { moyen } \\
\text { Mean } \\
\text { square }\end{array}$ & F \\
\cline { 2 - 4 } & 2,20663 & 5 & 0,44132 & $11,4++$ \\
Type de fromage (type of cheese) & 2,1338 & 55 & 0,03878 & \\
Résiduelle (residual) & 4,34001 & 60 & & \\
Totale (total) & & & \\
\hline
\end{tabular}

++ : Hypothèse nulle rejetée au niveau 0,01 .

++ : Null hypothesis rejected at a 0.01 level. 
dans le tableau 2 devraient être appliquées pour chaque type de fromage aux mesures fournies par l'appareil. Dans le cas contraire (calibrage unique de l'appareil pour tous les types de fromage) et en se basant sur le carré moyen total donné par l'analyse de variance, on obtient une précision d'estimation de l'extrait sec, quel que soit le type de fromage, égal à $\pm 0,54$ d'extrait sec pour $95 \%$ des échantillons examinés (écart-type des écarts de 0,27).

\section{c) Application au contrôle de l'humidité des fromages en cours d'égouttage}

$\mathrm{Au}$ cours de cinq fabrications différentes de fromage à pâte molle, des prélèvements de fromage ont été faits à $1 / 2,1,3,6,24$ et $48 \mathrm{~h}$ après moulage du caillé. Sur chaque échantillon la teneur

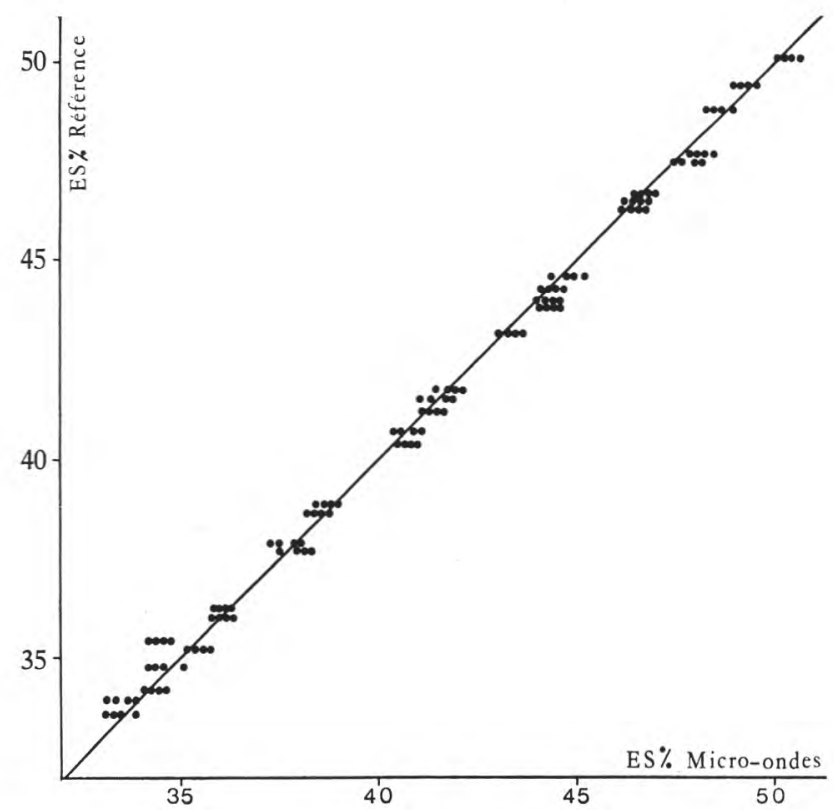

Fig. 1

Relation entre les teneurs en extrait sec de 30 échantillons de fromage à pâte molle en cours d'égouttage, mesurée par micro-ondes (4 déterminations par échantillon) et par la méthode de référence (moyenne de 3 déterminations par échantillon).

Relationship between the dry matter contents of 30 soft cheese samples during drainage, measured by microwave (4 replicates) and by reference (mean of 3 replicates) methods. 
en extrait sec a été déterminée en triple exemplaire par la méhode de référence et en quatre exemplaires à l'aide du four à micro-ondes.

Nous avons porté sur la figure 1 les valeurs individuelles trouvées par micro-ondes sur chaque échantillon en fonction des teneurs moyennes en extrait sec données par la méthode de référence. A partir de la régression des valeurs de référence sur chaque valeur individuelle de la méthode micro-ondes, on obtient un écart-type résiduel de $0,34 \%$ d'extrait $\mathrm{sec}$, indiquant qu'on peut estimer le taux d'extrait sec du fromage à partir d'une seule détermination au four à micro-ondes avec une précision de $\pm 0.68 \%$ d'extrait sec dans $95 \%$ des cas.

Ce dernier résultat illustre l'intérêt que présente l'utilisation d'un four à micro-ondes en fromagerie puisqu'il permet de mesurer l'extrait sec du fromage en cours d'égouttage avec une assez bonne précision $( \pm 0,68 \%$ d'extrait $\mathrm{sec})$ et ceci en 5 à 6 min environ, compte tenu du temps de préparation de l'échantillon.

\section{Remerciements}

Nous remercions la Société Anritsu pour le prêt de l'appareil et Isabelle Monneret pour sa collaboration technique.

\section{Bibliographie}

BigGs (D. A.) (1979). - Applications of infra-red instrumentation in cheese analysis. Proceedings from the Ist Biennal Marschall Int. Cheese Conf. Madison WI, Sept. 10-14.

Corrieu (G.), Lalande (M.) et Fissette (1982). - Corrélation entre la teneur en matière sèche du fromage frais maigre et sa viscosité apparente mesurée en ligne. Sciences des aliments, 2, 41-54.

Curnutte (B) (1980). - Principles of microwave radiation. J. Food Protec., 43, 618-624.

GrapPIN (R.) (1976). - Guide pour l'évaluation des méthodes d'analyses de routine. Le Lait, 56, 608-621.

GRAPPIN (R.) and JeUnet (R.) (1982). - Analytical performances of microwave oven and infrared methods for the determination of total milk solids. Brief communications. International Dairy Congress, Moscow, Vol. 1, 171-172.

Hamada (H.), Yoshino (M.), Shiga (K.), Takahashi (Y.), Nagai (A.) and Suga (N.) (1977). - Rapid determination of milk solids by microwave heater and electrobolance. J. Dairy Sci., 60, 1363-1367.

Multon (J. L.), Bizot (H.) et Martin (G.) (1981). - Eau (teneur, activité, absorption, propriétés fonctionnelles). Humidités relatives. Dans : Techniques d'analyse et de contrôle dans les industries agro-alimentaires. Tome 4 édité par Technique et Documentation et Apria, Paris. 
Pieper (H.), Suart Jr (J. A.) and Renwich (W.) (1977). - Microwave technique for rapid determination of moisture in cheese. Journal of the A.O.A.C., 60, 1392-1396.

Shanley (R. M.) and Jameson (G. W.) (1981). - A study of the rapid determination of moisture in cheese by microwave heating. Aust. J. Dairy Technol., 36, 107-109.

Takahashi (Y.), Nagai (A.), Suga (N.) and Chiba (J.) (1978). - Measurement of total milk solids by microwave heating. Journal of Microwave Power, 13, 167-171. 